\title{
El desarrollo de la conducta asertiva a estudiantes de nivel superior de Xilitla, S.L.P
}

\section{The development of assertive behavior to higher level students of Xilitla, S.L.P}

\author{
OLIVARES-BAZÁN, León Donizetty*†, INFANTE-ZAPUCHE, Eva y ZAPATA-PADILLA, Néstor \\ Juan
}

Universidad Autónoma de San Luis Potosí, Unidad Académica Multidisciplinaria Zona Huasteca Nombre, calle Romualdo del Campo No. 501, Frac. Rafael Curiel C.P. 79060, Ciudad Valles, S.L.P., México

ID $1^{\text {er }}$ Autor: León Donizetty, Olivares-Bazán / ORC ID: 0000-0002-2070-0229, CVU CONACYT ID: 1000662
ID $1^{\text {er }}$ Coautor: Eva Infante-Zapuche / ORC ID: 0000-0001-5667-3296, CVU CONACYT ID: 1000826
ID $2^{\text {do }}$ Coautor: Néstor Juan, Zapata-Padilla / ORC ID: 0000-0003-3367-3589, CVU CONACYT ID: 898332

DOI: $10.35429 /$ JPDL.2019.15.5.22.33

Recibido 29 de Marzo, 2019; Aceptado 30 Junio, 2019

\section{Resumen}

Objetivos: Diseñar un Curso-Taller para incrementar la asertividad en los alumnos de la Licenciatura de Gestión y Desarrollo Empresarial en el Instituto de Educación Superior Educadistanciaslp de Xilitla, S.L.P. Metodología: Se aplicó la investigación descriptiva para dar una explicación detallada de las características del objeto que se está evaluando. Con un enfoque cualitativo y cuantitativo. Además, se utilizó métodos (Histórico Lógico, Análisis - síntesis) teóricos, empíricos (Observación, Cuestionario, criterios de expertos) y matemáticos (Análisis porcentual y Grafico de barras). Contribución: Al integrar el Curso-Taller para incrementar la asertividad en los alumnos de la Licenciatura en Gestión y Desarrollo Empresarial se crea un precedente, al fomentar en el alumno la participación en clase e incrementar al mismo tiempo el conocimiento, de tal manera que egresen alumnos capaces de debatir sin temor alguno en el contexto laboral donde se desempeñen. Con el desarrollo del Curso-Taller para incrementar la asertividad en los alumnos, será más dinámica la clase, logrará mejorar los conocimientos y desarrollo, a la vez que adquirirá seguridad para expresar opiniones libremente, preparándolo para el desempeño laboral al terminar la Licenciatura, al mismo tiempo que el prestigio de la Institución crecerá al egresar personal altamente capacitado.

Comportamiento, Conducta asertiva, Liderazgo

\begin{abstract}
Objetive: Design a Course-Workshop to increase assertiveness in students of the Bachelor of Management and Business Development at the Institute of Higher Education Educadistanciaslp de Xilitla, S.L.P. Methodology: Descriptive research was applied to give a detailed explanation of the characteristics of the object being evaluated. With a qualitative and quantitative approach. In addition, theoretical, empirical (Observation, Questionnaire, expert criteria) and mathematical (Percent Analysis and Bar Graphs) methods (Historical - Logic, Analysis - synthesis) were used. Contribution: By integrating the Course-Workshop to increase assertiveness in students of the Bachelor of Business Management and Development, a precedent is created, by encouraging student participation in class and increasing knowledge at the same time, so that capable students graduate to debate without fear in the work context where they work. With the development of the Course-Workshop to increase assertiveness in students, the class will be more dynamic, will improve knowledge and development, while acquiring security to express opinions freely, preparing it for work performance at the end of the Degree, at at the same time that the prestige of the Institution will grow by graduating highly trained personnel.
\end{abstract}

Behaivor, Assertive behavior, Leadership

Citación: OLIVARES-BAZÁN, León Donizetty, INFANTE-ZAPUCHE, Eva y ZAPATA-PADILLA, Néstor Juan. El desarrollo de la conducta asertiva a estudiantes de nivel superior de Xilitla, S.L.P. Revista de Filosofía y Cotidianidad. 2019, 5-15: 22-33

\footnotetext{
* Correspondencia del Autor (donizetty.bazan@uaslp.mx)

$\dagger$ Investigador contribuyendo como primer Autor.
} 


\section{Introducción}

En el artículo tercero de la Constitución Mexicana se establece la obligatoriedad de la Educación Básica y Media Superior en toda la República Mexicana y dice en el párrafo segundo que la educación que imparta el estado tenderá a desarrollar armónicamente todas las facultades del ser humano y fomentar en él, a la vez, el amor a la patria, el respeto a los derechos humanos y la conciencia de la solidaridad internacional, en la independencia y en la justicia.

Con el artículo mencionado se puede entender que el alumno que egresó del nivel Medio Superior y que ingresó al Nivel Superior, en este caso Licenciatura, debió estar capacitado para desarrollarse dentro del salón de clases, demostrando compañerismo e iniciativa tanto de participación como de técnicas de estudio para obtener el máximo desempeño y aprendizaje de las materias que se imparten dentro del estudio profesional.

Sin embargo, si se contextualiza en el ámbito educativo sobre todo en áreas geográficas donde los usos y costumbres de la región tienen un impacto determinante en la forma en que se desempeñó el alumno y la forma de vivir de la sociedad en general, debió haber representado un reto para el docente lograr el cambio de conducta en el grupo escolar al que se impartió clase, habiendo tenido que desarrollar estrategias especiales que coadyuvaran a superar las barreras que impone el contexto, para haber dado lugar a habilidades que permitieran desarrollar en el alumno las facultades a las que hace referencia el artículo tercero.

Esta investigación estableció como objetivo el diseño de un Curso-Taller para incrementar la asertividad en los alumnos de la Licenciatura de Gestión y Desarrollo Empresarial, tomando como población 16 alumnos que conforman el total de los estudiantes que cursan esta licenciatura.

Se establecieron cinco preguntas científicas: ¿Cómo se conceptualiza en la literatura existente, la asertividad en los estudiantes del nivel superior? ¿Cuáles son las características situacionales que prevalecen respecto de la conducta asertiva en los alumnos de la Licenciatura en Gestión y Desarrollo Empresarial del Instituto de Educación Superior Educadistanciaslp, de Xilitla, S.L.P.?
¿Cómo diseñar un Curso-Taller que ayude a incrementar la asertividad en los alumnos de la Licenciatura de Gestión y Desarrollo Empresarial del Instituto de Educación Superior Educadistanciaslp en Xilitla, S.L.P.? ¿Cómo evaluar de manera preliminar el Curso-Taller propuesto para incrementar la asertividad en los alumnos de la Licenciatura en Gestión y Desarrollo Empresarial del Instituto de Educación Superior Educadistanciaslp, de Xilitla, S.L.P.?

Con el desarrollo del Curso-Taller para incrementar la asertividad en los alumnos, será más dinámica la clase, logrará mejorar los conocimientos y desarrollo, a la vez que adquirirá seguridad para expresar opiniones libremente, preparándolo para el desempeño laboral al terminar la Licenciatura, al mismo tiempo que el prestigio de la Institución crecerá al egresar personal altamente capacitado.

El articulo está formado por tres subtemas: en el primer subtema abarcara la parte de la Fundamentación teórica sobre la asertividad en alumnos de Nivel Superior. El segundo subtema se presentará el diagnóstico sobre la asertividad de los alumnos. La tercera parte será la propuesta del curso-taller cuyo fin es la incrementar la asertividad a los estudiantes y por ultimo las conclusiones generales de la investigación realizada así como a su vez unas recomendaciones sobre el uso de la propuesta.

\section{Fundamentación teórica sobre la asertividad en alumnos de nivel superior}

La forma de educar a los hijos en algunas regiones de la república tiene características particulares, entre ellos dogmas religiosos, familiares y culturales, que van heredándose de generación en generación y que están arraigadas y es difícil cambiar en corto tiempo.

En este trabajo se definen solo tres tipos de conducta, la conducta inhibida, la conducta agresiva y la conducta asertiva: Roca Elia, (2003) define la conducta inhibida como sumisión, pasividad, retraimiento, tendencia a adaptarse excesivamente a reglas externas o a deseos de los demás, sin tener bastante en cuenta los propios intereses, sentimientos, derechos, opiniones y deseos. Esta conducta es generalmente encontrada donde los hijos deben cumplir una serie de reglas sin derecho a opinión para no ser excluidos del medio en el que se desarrollan.

OLIVARES-BAZÁN, León Donizetty, INFANTE-ZAPUCHE, Eva y ZAPATA-PADILLA, Néstor Juan. El desarrollo de la conducta asertiva a estudiantes de nivel superior de Xilitla, S.L.P. Revista de Filosofía y Cotidianidad. 2019 
La conducta agresiva la define Elia Roca, (2003) como: conducta no-asertiva, opuesta a la inhibición, no respeta los derechos, sentimientos e intereses de los demás en su forma más extrema incluye: ofenderlos, provocarlos o atacarlos. De esta conducta se deriva la conducta pasivo-agresiva: es la de quien no se atreve a mostrarse directamente agresivo, pero lo hace indirectamente. Esta derivación es muy común encontrarla en cualquier contexto social logrando tensionar el ambiente de convivencia.

Sobre la conducta asertiva, dice Elia Roca, (2003) que una persona tiene una conducta asertiva cuando defiende los propios intereses, se expresa opinando libremente y no permite que los demás se aprovechen de ella; al mismo tiempo, es considerada con la forma de pensar y de sentir de los demás. Esta conducta es el término medio entre conducta inhibida y conducta agresiva. La conducta asertiva tiene como características ciertos derechos, como ejemplo: en lugar de pensar que se deben acatar los puntos de vista de los demás, se debe ejercer el derecho de tener una opinión propia, o en otro ejemplo, no se debe creer que cometer errores es vergonzoso, el ser humano tiene derecho a cometer errores.

La cultura no-asertiva tiende a fomentarse cuando se observa la manera en que se conducen personas no-asertivas, o cuando se elogian las conductas no asertivas, o por ejemplo con la crítica se castigan las conductas asertivas, muy común en los grupos de personas. Este tipo de situaciones hace que sea más difícil adoptar una conducta asertiva, sobre todo si no hay alguien que asesore, en este caso alumnos, sobre cómo adoptar una conducta asertiva.

Algunas definiciones de asertividad: León, Rodríguez, Ferrel, Ceballos, (2009). Definen la asertividad como la habilidad del individuo para expresar las propias limitaciones, sentimientos, opiniones, deseos, derechos, para dar y recibir alabanzas, hacer peticiones $\mathrm{y}$ manejar la crítica. Güel, (2005). Asertividad: Es la expresión de los propios intereses, creencias, opiniones y deseos de manera honrada, tranquila, sin sentimiento de culpa y sin perjudicar ni agredir los deseos, intereses o derechos de los otros.
Las características de las conductas:

La conducta Pasiva la describe Trejo (2006) con las siguientes características:

Conducta General. Actúa con la esperanza de que los demás adivinen los deseos. La apariencia es de inseguridad.

Verbalmente. $\mathrm{Se}$ disculpa constantemente; da mensajes indirectos; habla con rodeos; no encuentra palabras adecuadas; no dice lo que quiere decir; habla mucho para clarificar su comunicación; no dice nada por miedo o vergüenza; se humilla a sí mismo.

Voz. Débil, temblorosa; volumen bajo

Mirada. Evita el contacto visual; ojos caídos y llorosos.

Postura. Agachada; mueve la cabeza en forma afirmativa constantemente.

Manos. Temblorosas y sudorosas

La conducta Agresiva la describe Trejo (2006) con las siguientes características:

Conducta General. Exagera para demostrar la superioridad. Refleja agresividad.

Verbalmente. Exige; usa palabras altisonantes. Hace acusaciones; impone la opinión; se comunica a base de mandatos; usa mensaje "TÚ"; habla mucho para no ser contrariado y llamar la atención. Se sobrestima; habla solamente de sí mismo.

Voz. Fuerte; con frecuencia grita; voz fría y autoritaria.

Mirada. Sin expresión; fija, penetrante y orgullosa.

Postura. Rígida; desafiante y soberbia.

Manos. Usa el dedo acusatorio; movimientos rechazantes o aprobatorios

Brown (1980) define la conducta asertiva como la conducta que ocurre en un contexto interpersonal que se interesa principalmente por la adquisición de refuerzo personal y que minimiza la pérdida de refuerzo por parte del (los) receptor(es) de la(s) conducta(s). 
De acuerdo con Caballo (1983) la conducta asertiva es aquella conducta que expresa los sentimientos y pensamientos de un individuo de una manera honesta sin herir a los demás, y que normalmente alcanza el objetivo.

La conducta Asertiva la describe Trejo (2006) con las siguientes características:

Conducta General. Actúa con naturalidad; escucha atentamente.

Verbalmente. Expresa lo que quiere; expresa sentimientos; habla objetivamente; usa mensajes "YO"; habla cuando tiene algo que decir; habla bien de sí mismo si es necesario o conveniente; la comunicación es directa. modulada.

Voz. Firme; calurosa; relajada; bien

Mirada. Ve a los ojos; mirada franca; ojos expresivos. tranquila.

Postura. Bien balanceada; relajada y

Manos. Movimientos relajados, naturales y acogedores.

En las habilidades alternativas a la agresión: Acosta (2003) dice que en cualquier momento o circunstancia se quiere satisfacer alguna de las necesidades personales y alcanzar el logro de lo que se desea. Pero no siempre se consigue, los intereses personales pueden entrar en conflicto con los de otras personas y puede que, entonces, sea imposible alcanzarlos.

En esas circunstancias, no es extraño que se sienta el impulso de arremeter contra otra u otras personas por entender que ellas son las causantes de que no se logren las metas. Algunas personas argumentan que cuando son agresivos lo que pretenden es defender los derechos. Eso puede ser cierto. Pero, es importante darse cuenta que la valía personal y los derechos pueden defenderse con comportamientos no agresivos.

Las habilidades alternativas a la agresión: a) No entrar en peleas; b) Emplear autocontrol; c) Responder a las bromas; d) Convencer a los demás e) Negociar; f) Compartir algo; g) Ayudar a los demás.

\begin{tabular}{|c|c|}
\hline Legítimos derechos & $\begin{array}{l}\text { Suposiciones tradicionales } \\
\text { erróneas }\end{array}$ \\
\hline $\begin{array}{l}\text { Algunas veces, se tiene } \\
\text { derecho a ser el primero. }\end{array}$ & $\begin{array}{l}\text { Es ser egoísta, anteponer las } \\
\text { necesidades propias a las de los } \\
\text { demás. }\end{array}$ \\
\hline $\begin{array}{l}\text { Se tiene derecho a } \\
\text { cometer errores. }\end{array}$ & $\begin{array}{l}\text { Es vergonzoso cometer errores. Hay } \\
\text { que tener una respuesta adecuada } \\
\text { para cada ocasión. }\end{array}$ \\
\hline $\begin{array}{l}\text { Se tiene derecho a ser } \\
\text { juez último de los } \\
\text { sentimientos y aceptarlos } \\
\text { como válidos. }\end{array}$ & $\begin{array}{l}\text { Si no puede convencer a los demás } \\
\text { de que los sentimientos son } \\
\text { razonables, debe ser que se está } \\
\text { equivocado o que se está volviendo } \\
\text { loco. }\end{array}$ \\
\hline $\begin{array}{l}\text { Se tiene derecho a tener } \\
\text { las propias opiniones y } \\
\text { convencimientos. }\end{array}$ & $\begin{array}{l}\text { Hay que respetar los puntos de vista } \\
\text { de los demás, especialmente si } \\
\text { desempeñan algún cargo de } \\
\text { autoridad. Guardarse las diferencias } \\
\text { de opinión para uno mismo; } \\
\text { escuchar y aprender. }\end{array}$ \\
\hline $\begin{array}{l}\text { Se tiene derecho a } \\
\text { cambiar de idea o de } \\
\text { línea de acción. }\end{array}$ & $\begin{array}{l}\text { Hay que intentar ser siempre lógico } \\
\text { y consecuente. }\end{array}$ \\
\hline $\begin{array}{l}\text { Se tiene derecho a la } \\
\text { crítica y a protestar por } \\
\text { un trato injusto. }\end{array}$ & $\begin{array}{l}\text { Hay que ser flexible y adaptarse. } \\
\text { Cada uno tiene sus motivos para } \\
\text { hacer las cosas y no es de buena } \\
\text { educación interrogar a la gente. }\end{array}$ \\
\hline $\begin{array}{l}\text { Se tiene derecho a } \\
\text { interrumpir para pedir } \\
\text { una aclaración }\end{array}$ & $\begin{array}{l}\text { No hay que interrumpir nunca a la } \\
\text { gente. Hacer preguntas denota } \\
\text { estupidez. }\end{array}$ \\
\hline $\begin{array}{l}\text { Se tiene perfecto derecho } \\
\text { a intentar un cambio. }\end{array}$ & $\begin{array}{l}\text { Las cosas podrían ser aún peores de } \\
\text { lo que son. No hay que tentar a la } \\
\text { suerte. }\end{array}$ \\
\hline $\begin{array}{l}\text { Se tiene derecho a pedir } \\
\text { ayuda o apoyo } \\
\text { emocional. }\end{array}$ & $\begin{array}{l}\text { No hay que hacer perder a los demás } \\
\text { su valioso tiempo con los problemas } \\
\text { propios }\end{array}$ \\
\hline $\begin{array}{l}\text { Se tiene derecho a sentir } \\
\text { y expresar el dolor. }\end{array}$ & $\begin{array}{l}\text { A la gente no le gusta escuchar que } \\
\text { una persona se encuentra mal, así } \\
\text { que es mejor guardárselo para sí. }\end{array}$ \\
\hline $\begin{array}{l}\text { Se tiene derecho a } \\
\text { ignorar los consejos de } \\
\text { los demás. }\end{array}$ & $\begin{array}{l}\text { Cuando alguien se molesta en dar un } \\
\text { consejo, es mejor tomarlo } \\
\text { seriamente en cuenta, porque suele } \\
\text { tener razón. }\end{array}$ \\
\hline $\begin{array}{l}\text { Se tiene derecho a recibir } \\
\text { el reconocimiento formal } \\
\text { por un trabajo bien } \\
\text { hecho. }\end{array}$ & $\begin{array}{l}\text { La satisfacción de saber que se ha } \\
\text { hecho algo bien es la mejor } \\
\text { recompensa. A la gente no le gustan } \\
\text { los alardes, la gente que triunfa, en el } \\
\text { fondo cae mal y es envidiada. Hay } \\
\text { que ser humilde ante los halagos. }\end{array}$ \\
\hline $\begin{array}{l}\text { Se tiene derecho a decir } \\
\text { "no". }\end{array}$ & $\begin{array}{l}\text { Hay que intentar adaptarse siempre a } \\
\text { los demás, de lo contrario no se } \\
\text { encuentran cuando se necesitan. }\end{array}$ \\
\hline $\begin{array}{l}\text { Se tiene derecho a estar } \\
\text { solo aún cuando los } \\
\text { demás deseen } \\
\text { compañía. }\end{array}$ & $\begin{array}{l}\text { No hay que ser antisocial. Si dices } \\
\text { que prefieres estar solo, los demás } \\
\text { pensarán que no te gustan. }\end{array}$ \\
\hline $\begin{array}{l}\text { Se tiene derecho a no } \\
\text { justificarse ante los } \\
\text { demás. }\end{array}$ & $\begin{array}{l}\text { Hay que tener siempre una buena } \\
\text { razón para todo lo que se siente y se } \\
\text { hace. }\end{array}$ \\
\hline $\begin{array}{l}\text { Se tiene derecho a no } \\
\text { responsabilizarse de los } \\
\text { problemas de los demás. }\end{array}$ & $\begin{array}{l}\text { Cuando alguien tiene un problema, } \\
\text { hay que ayudarle. }\end{array}$ \\
\hline $\begin{array}{l}\text { Se tiene derecho a no } \\
\text { anticiparse a las } \\
\text { necesidades y deseos de } \\
\text { los demás. }\end{array}$ & $\begin{array}{l}\text { Hay que ser sensible a las } \\
\text { necesidades y deseos de los demás, } \\
\text { aún cuando estos sean incapaces de } \\
\text { demostrarlos. }\end{array}$ \\
\hline $\begin{array}{l}\text { Se tiene derecho a no } \\
\text { estar pendiente de la } \\
\text { buena voluntad de los } \\
\text { demás. }\end{array}$ & $\begin{array}{l}\text { Es una buena política intentar ver } \\
\text { siempre el lado bueno de la gente. }\end{array}$ \\
\hline $\begin{array}{l}\text { Se tiene derecho a } \\
\text { responder o a no hacerlo }\end{array}$ & $\begin{array}{l}\text { No está bien quitarse a la gente de } \\
\text { encima; si alguien hace una } \\
\text { pregunta, hay que darle siempre una } \\
\text { respuesta. }\end{array}$ \\
\hline
\end{tabular}

Tabla 1 Derechos Asertivos comparados con las suposiciones erróneas

Fuente: Diez Gutiérrez, (2006) 
Trejo, (2006). Las personas asertivas conocen los propios derechos y los defienden, respetando a los demás, es decir, no van a ganar, sino a llegar a un acuerdo.

\section{Comportamiento externo.}

- Habla fluida, seguridad, ni bloqueos, ni muletillas, contacto ocular directo, pero no desafiante, relajación corporal, comodidad postural.

- Expresión de sentimientos tanto positivos como negativos, defensa sin agresión, honestidad, capacidad de hablar de propios gustos e intereses, capacidad de discrepar abiertamente, capacidad de pedir aclaraciones, decir no, saber aceptar errores.

Conocen y creen en unos derechos para sí y para los demás.

Las convicciones son en la mayoría racionales, la manera de pensar es más racional, actuando de forma más adaptativa a la vida y circunstancias.

Sentimientos o emociones:

Buena autoestima, no se sienten inferiores ni superiores a los demás, satisfacción en las relaciones, respeto por uno mismo.

- $\quad$ Sensación de control emocional.

También en este caso, la conducta asertiva tendrá unas consecuencias en el entorno y la conducta de los demás:

- $\quad$ Frenan o desarman a la persona que les ataque

- Aclaran equívocos

- Los demás se sienten respetados y valorados

- La persona asertiva suele ser considerada "buena", pero no "tonta

El problema de la asertividad en la Educación Superior caso en Argentina.

González, Cadoche, Prendes (2005) realizaron la investigación: La asertividad en alumnos universitarios en la Universidad Nacional del Litoral, en la Facultad de Ciencias Universitarias en Argentina. Se extrajo lo siguiente:
El objetivo fue la de Indagar, diagnósticamente, las competencias de asertividad de los alumnos ingresantes a la carrera de Medicina Veterinaria. Cohorte, (2007)

Para realizar esta evaluación, se utilizó un test, de "tendencia de asertividad", presentado por Pérez Pincheira (2004). Participaron de este ensayo un grupo de 146 alumnos de primer año de la carrera Medicina Veterinaria, todos ellos ingresantes del año 2007.

Los resultados fueron los siguientes:

- $\quad$ El $20 \%$ de los alumnos manifiesta inseguridad y baja autoestima, dado que afirman que suelen evitar contactos sociales por temor a hacer o decir algo inadecuado.

- Un $93 \%$ prefiere eludir situaciones problemáticas con otras personas antes que afrontarlas.

- $\quad$ El $50 \%$ no se cree capaz de enfrentar desafíos donde deban respetar y exigir respeto de los demás, siendo esto un llamado de atención ya que, debido al contexto antes mencionado de estos jóvenes, deberán ahora autogestionar la vida, tomar decisiones $\mathrm{y}$ enfrentar diversas situaciones.

- $\quad$ El $95 \%$ responde que conoce pocas personas con las que pueda sentirse relajado y pasarlo bien; es el adjetivo calificativo pocas lo que llama la atención.

El $68 \%$ de los alumnos parece mostrar reservas en la amistad frente al dinero, ya que afirma que dudarían en pedir dinero prestado a un amigo o en recordarle alguna deuda monetaria del otro para con él. Al parecer la confianza se pierde frente a una deuda económica.

Por otra parte se percibe la tendencia hacia una actitud asertiva en las siguientes observaciones:

- Sólo el $1 \%$ manifiesta no sentirse confiado para contradecir la opinión de alguien a quien respetan.

Un $100 \%$ afirma que cada persona debe defender sus derechos. 
Se concluye de las observaciones realizadas que, en muchos aspectos, los alumnos poseen un comportamiento inhibido o pasivo con una leve tendencia a la asertividad.

\section{Comparación entre México - Cuba}

En el estudio, Asertividad: un estudio comparativo entre estudiantes de México y Cuba, se extrajo lo siguiente:

Flores, Lainé (2009) Con una muestra de 426 universitarios de los cuales $50.5 \%$ (215) fueron de Mérida, Méx., y 49.5\% (211) de la Ciudad de la Habana, Cuba.

\begin{tabular}{|l|c|c|c|c|}
\hline $\begin{array}{c}\text { Factores de la Escala } \\
\text { Multidimensional de } \\
\text { Asertividad }\end{array}$ & MEX & CUB & & \\
\hline \multicolumn{2}{|c|}{ PAÍS } & Frob. \\
\hline Asertividad Indirecta & 2.45 & 2.13 & 16.19 & $0.000^{* * *}$ \\
No Asertividad & 2.63 & 2.26 & 24.50 & $0.000^{* * *}$ \\
Asertividad & 3.72 & 4.14 & 58.24 & $0.000^{* * *}$ \\
\hline
\end{tabular}

Tabla 2 Factores de la Escala Multidimensional de Asertividad en el estudio comparativo México - Cuba.

Los resultados muestran diferencias significativas en los tres factores (Ver tabla No. 2) de la Escala Multidimensional en Asertividad entre los estudiantes de México y Cuba, aunque el patrón de respuesta fue muy similar, se observó que en los factores de asertividad indirecta $y$ no asertividad los estudiantes mexicanos son los que obtuvieron las medias más altas, es importante señalar que éstas están por debajo de la media teórica. Por el contrario, los estudiantes cubanos son los que obtienen la media más alta en el factor de asertividad, a pesar de que para ambos, las medias obtenidas estuvieron por arriba de la media teórica.

Hallazgos matizados por la cultura, puesto que aunque ambos países son colectivistas, desde el enfoque etnopsicológico, la personalidad del mexicano se ha explicado a partir de las Premisas Histórico-SocioCulturales destacando en ellas, la obediencia afiliativa y del estilo automodificador, DíazGuerrero, (1994) por lo que no es de extrañar que los mexicanos obtengan puntuaciones menos altas en asertividad. Flores y Díaz-Loving, (2004); Flores, Díaz-Loving, Padilla y (Castillo, en prensa).

\section{Diagnóstico sobre la asertividad de los alumnos}

Los instrumentos utilizados fueron constituidos por encuestas a los alumnos que cursan la carrera de Gestión y Desarrollo Empresarial del Instituto de Educación Superior Educadistanciaslp y al personal docente que imparte en esta Licenciatura, así como la aplicación del instrumento de la observación de clase. Los resultados obtenidos se analizaron y representaron utilizando gráficas de barras y pastel, mostrando los resultados en cantidad porcentuales.

La información obtenida se analizó de acuerdo con el método de triangulación para obtener respuesta a la pregunta científica: ¿Cuáles son las características situacionales que prevalecen respecto de la conducta asertiva en los alumnos de la Licenciatura en Gestión y Desarrollo Empresarial del Instituto Educadistanciaslp, de Xilitla, S.L.P.?

La población estudiantil de la Licenciatura de Gestión y Desarrollo Empresarial del Instituto de Educación Superior Educadistanciaslp, cuyo total de estudiantes suma 16 y para la investigación se ha utilizado el universo completo. El instrumento que se utilizó fue la encuesta a alumnos, donde se obtuvo los siguientes resultados:

1.- Cuando estás en clase con algún tema ¿participas libremente?

$69 \%$ a veces; $31 \%$ siempre

2.- ¿Cuándo estás en clase, expresas tu opinión consciente de que puedes cometer errores?

$50 \%$ a veces; $44 \%$ siempre; $6 \%$ de vez en cuando

3.- Si el maestro te hace una pregunta sobre algo de la clase y no sabes la respuesta, ¿Te sientes incómodo?

$44 \%$ de vez en cuando; $25 \%$ a veces; $12 \%$ nunca

4.- Si por alguna circunstancia, no te enteras de algo importante de la clase, ¿Pides que te informen en cuanto te das cuenta?

$75 \%$ siempre; $19 \%$ a veces; $6 \%$ nunca

5.- Cuando un compañero de clase, te pide que hagas algo en lo que no estás de acuerdo, ¿De todas maneras lo haces?

$50 \%$ nunca; $38 \%$ de vez en cuando; $12 \%$ a veces 
6.- Cuando un compañero de clase, te pide ayuda en algo que no sabes o no conoces, ¿Le dices abiertamente que no puedes ayudarle?

$50 \%$ siempre; $19 \%$ a veces; $12 \%$ de vez en cuando; $19 \%$ nunca

7.- Si tienes un evento en la escuela en el que se requiere la participación individual y tú estás de acuerdo, ¿participas en tal evento, aunque la mayoría de tu grupo no esté de acuerdo?

$37 \%$ a veces; $31 \%$ siempre; $13 \%$ nunca; $10 \%$ de vez en cuando

8.- $¿$ Te apuras en sacar buenas evaluaciones para no perder tu prestigio de "bueno, responsable o admirable?

$50 \%$ a veces; $25 \%$ siempre; $19 \%$ nunca; $6 \%$ de vez en cuando

9.- Cuando presentas un examen, sientes que es muy importante para ti, ¿tener la más alta calificación en comparación con tus compañeros de clase?

$44 \%$ a veces; $25 \%$ siempre; $25 \%$ nunca; $6 \%$ de vez en cuando

10.- Si el maestro hace alguna pregunta, y tienes la respuesta, ¿Te molesta que otro compañero dé antes la respuesta?

$56 \%$ nunca; $25 \%$ de vez en cuando; $19 \%$ a veces

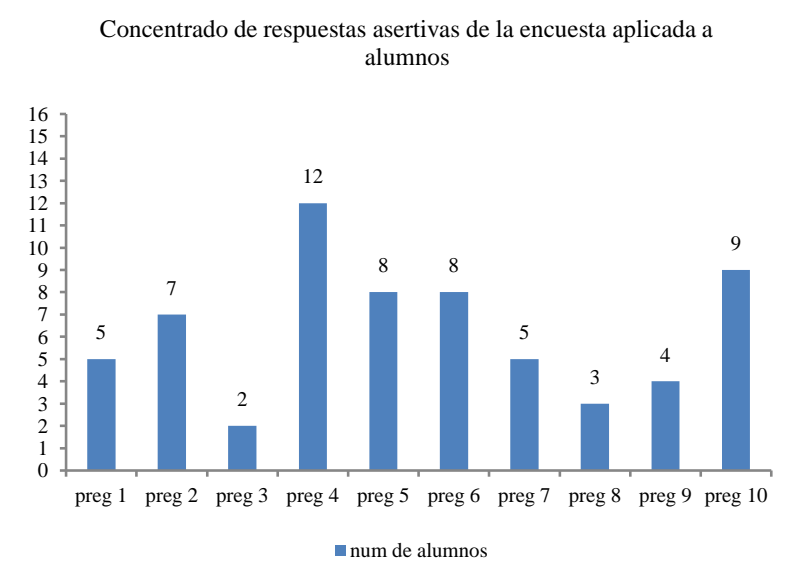

Grafico 1 Concentrado respuestas asertivas en encuesta aplicada a alumnos

Fuente: Elaboración Propia

En el concentrado de respuestas asertivas de este instrumento de investigación EAMAPLE-EIZ/2014, se puede observar que existe un número considerable de alumnos que generalmente no presentan una conducta asertiva con respecto a sus clases.
Se aplicó el instrumento EPD-MAPLEEIZ/2014 encuesta a docentes, se obtuvieron los siguientes resultados:

1.- ¿Cómo valora la actitud de los alumnos en su participación libre?

$67 \%$ muy bien; $33 \%$ bien

2.- ¿Cómo valora la seguridad que muestran los alumnos cuando expresan su opinión, a pesar que lo expresado sea incorrecto?

$34 \%$ muy bien; $33 \%$ bien; 33\% regular

3.- ¿Cómo valora la reacción de los alumnos cuando les pregunta directamente algo que desconocen, del tema que se esté tratando en clase?

$34 \%$ muy bien; $33 \%$ bien; 33\% regular

4.- ¿Cómo valora la actitud de los alumnos que no tienen información completa sobre algo de la clase?

$34 \%$ bien; $33 \%$ regular; $33 \%$ mal

5.- ¿Cómo valora la actitud de los alumnos en las decisiones erróneas del líder del grupo?

$67 \%$ bien; $33 \%$ mal

6.- ¿Cómo valora entre alumnos la colaboración entre pares al aplicar una estrategia de trabajo? $67 \%$ muy bien; $33 \%$ bien

7.- ¿Cómo valora la participación individual de los alumnos en algún proyecto de colaboración? $67 \%$ muy bien; $33 \%$ bien

8.- ¿Cómo valora la actitud de los alumnos al compartir notas fuera de clase con los demás compañeros?

$67 \%$ muy bien; $33 \%$ bien

9.- ¿Cómo valora la convivencia de los alumnos que tienen las mejores notas, con sus demás compañeros?

$33 \%$ muy bien; $67 \%$ bien

10.- ¿Cómo valora la reacción de los alumnos que van a dar alguna respuesta a algo que se les pregunta y otro alumno la emite antes?

$100 \%$

$\mathrm{Al}$ analizar los resultados obtenidos en la aplicación de los instrumentos se observan algunas similitudes y discrepancias en la información obtenida: 
En el análisis del instrumento de encuesta a alumnos, se observa que existe buena comunicación entre pares, sin embargo, al cuestionar sobre las actitudes asertivas que deben mostrar en el salón de clase, sólo una parte del alumnado la ha desarrollado, habiendo similitud en las respuestas del cuestionario a docentes.

De acuerdo con las gráficas del cuestionario a docentes se observa que los docentes consideran que una parte del alumnado desarrolla en clase las conductas de iniciativa en la participación de clase. En la observación de clase también se observó que una parte del alumnado ha desarrollado la iniciativa de participación en clase.

Existe discrepancia en algunas actitudes de los alumnos como en la participación individual en eventos, donde solamente el $31 \%$ muestra una conducta asertiva de acuerdo con la encuesta a alumnos y en la encuesta a docentes el $67 \%$ considera que los alumnos tienen una actitud colaborativa.

Se observó que una gran parte del alumnado no ha desarrollado la conducta asertiva dentro del salón de clase, mermando de ésta manera la oportunidad de obtener el máximo conocimiento de las materias que se les imparten.

\section{Propuesta del curso-taller para incrementar la asertividad}

En esta propuesta de solución se presenta a los estudiantes los conocimientos necesarios sobre la asertividad y a través de ejercicios dinámicos y con el apoyo de videos el alumno se apropiará de las nuevas conductas necesarias para el mejoramiento del desempeño escolar, favoreciendo al mismo tiempo el desarrollo de la iniciativa y la habilidad de liderazgo.

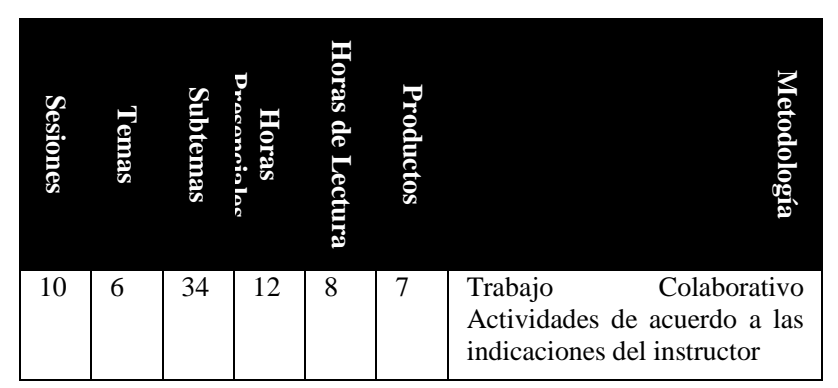

Tabla 3 Características generales de la propuesta Fuente: Elaboración Propia
Esta es una propuesta formativa constituida por 10 sesiones, mismas que deberán cubrirse en un tiempo de $20 \mathrm{hrs}$. En el desarrollo, el alumno analizará los contenidos, realizará actividades y se estudiará a sí mismo, para que de esta manera se obtengan productos específicos que beneficien el desarrollo de clase y el desempeño del alumno.

En la primera sesión se abordan los conceptos esenciales y conocimientos esenciales del tema, que ayudarán al alumno a entender cuál debe ser su desempeño dentro del aula y también se motive a participar en la misma, enriqueciendo el desarrollo de clase y mejorando su preparación académica.

En la segunda, tercera, cuarta, quinta, sexta, séptima, se exploran y abordan los derechos, principios básicos, lenguaje verbal y no verbal de la asertividad y se estudiará la persona asertiva así como los diferentes tipos de conducta que se pueden presentar.

En la octava sesión se sugiere la proyección de una película para que el alumno analice las conductas que se presentan en la misma, en la novena sesión se llevará a cabo un ejercicio de retroalimentación, resolución de dudas y reforzamiento de los puntos que el instructor considere que hayan quedado débiles en el transcurso del Curso-Taller, en la décima sesión se sugiere la aplicación de un test de evaluación con la finalidad de conocer el grado de asimilación y cambio de conducta de los alumnos que participaron en el Curso-Taller.

La metodología del curso es el siguiente:

En la primera etapa del Curso - Taller para incrementar la asertividad en los alumnos de la Licenciatura de Gestión y Desarrollo Empresarial del Instituto de Educación Superior Educadistanciaslp de Xilitla, S.L.P., los contenidos son abordados en la línea de "teoría - práctica" como eje metodológico, por lo tanto todas las actividades están encaminadas en esa relación. En cada tema abordado, se contempla una exposición teórica por parte del instructor acompañada con material de apoyo como videos y diapositivas, interactuando con el grupo, haciendo comparación con el desarrollo normal de una clase. Se realizan actividades utilizando técnicas que ayuden a los alumnos a conocer las conductas asertivas, ayudándolos para la facilidad de adaptación a las mismas. 
La segunda etapa del Curso-Taller se efectúa a través de ejercicios una valoración de las nuevas conductas adquiridas por los alumnos, se corrigen aspectos y se hace retroalimentación a los participantes retomando los conceptos más importantes y reafirmando los puntos que el instructor considere que hayan quedado débiles.

En la tercera etapa se aplicará un test para conocer el grado de adquisición de habilidades asertivas de los participantes en el Curso - Taller

\section{Recomendaciones para el uso de la propuesta}

Se recomienda que esta propuesta se aplique a toda la comunidad estudiantil del instituto, para que todos tengan oportunidad de beneficiarse con la práctica de éste taller.

Se recomienda que ya que el docente tiene un papel muy importante dentro del salón de clases se les invite a participar en este taller, con la finalidad de que motiven a los alumnos dentro del salón de clase a practicar las conductas asertivas.

Se recomienda que los docentes de cada materia hagan su planeación tomando en cuenta las habilidades asertivas que el alumno debe desarrollar coadyuvando en el proyecto de la institución, de crear líderes asertivos.

Se recomienda aplicar esta propuesta en otro nivel escolar para incrementar la asertividad en los alumnos.

\section{Conclusión}

Al desarrollar esta investigación, la idea principal es apoyar a los alumnos de la Licenciatura en Gestión y Desarrollo Empresarial a desarrollar habilidades asertivas, para mejorar el desempeño de la clase, que el alumno tenga iniciativa propia para expresar su opinión de acuerdo a los temas que se estén llevando a cabo, y que no sea necesario esperar que el docente le pregunte para opinar, sobre todo porque entre los alumnos que son muy callados, la mayoría tienen una capacidad de análisis y de interpretación muy alta, y que el docente la descubre hasta que dirige a esas personas sus cuestionamientos.
Se ha investigado varios autores, que hablan de la necesidad de desarrollar habilidades asertivas que ayudan a que las personas actúen respetándose y respetando a los demás, a la vez que las personas aprenden a aceptarse tal como son y a fomentar su desarrollo personal. Las actitudes asertivas permiten que las personas tengan la iniciativa necesaria coadyuvando al desarrollo de la habilidad de liderazgo y gestión.

Una vez aplicados los instrumentos de la investigación, graficados y analizados, se concluye que los docentes tienen un buen desempeño en la impartición de clase, utilizan estrategias y motivan a sus alumnos, pero una gran mayoría de alumnos no demuestra iniciativa de participación en clase.

Esta falta de iniciativa no se ve que sea apatía, sino, que se deduce que es un poco de inseguridad o temor a equivocarse. Es necesario apoyar a los alumnos para que desarrollen habilidades de iniciativa y liderazgo y se cumpla eficientemente el perfil del egresado programado.

La implementación del Curso-Taller pone de manifiesto la importancia que el Instituto de Educación Superior Educadistanciaslp tiene en el mejoramiento del aprovechamiento de sus alumnos. Pone en práctica nuevas ideas y comportamientos dentro del salón de clase, liberando a los alumnos de conductas no asertivas para insertarlos en un nuevo mundo empresarial, donde es necesaria la iniciativa y los conocimientos para generar nuevos líderes y gestionadores en la actividad económica.

En la idea de que los egresados de la Licenciatura en Gestión y Desarrollo Empresarial deben ser líderes positivos con iniciativa, sus conductas deben ser asertivas para que guíen por buen sendero en el ramo empresarial y logren eficiente y eficazmente el objetivo del perfil del egresado de la Licenciatura en Gestión y Desarrollo Empresarial del Instituto Educadistanciaslp. 


\section{Anexos}

Sesión No. 1. Conceptos y teoría básicos sobre asertividad

1.1 Concepto de Asertividad

1.2 Concepto de Empatía

1.3 Concepto de Habilidades Sociales

1.4 Ventajas de la asertividad

Sesión No. 2. Principios básicos de la asertividad

2.1 Respeto por los demás y por uno mismo

2.2 Ser directo

2.3 Ser Honesto

2.4 Ser Apropiado

Sesión No. 3. Lenguaje Verbal y no Verbal

3.1 Gestos con rostro

3.2 Gestos con las manos

3.3 Gestos con el cuerpo

3.4 La voz

Sesión No. 4 Derechos Asertivos

4.1 Derecho a ser el primero

4.2 Derecho a cometer errores

4.3 Derecho a ser juez último de los sentimientos y aceptarlos como válidos

4.4 Derecho a tener las propias opiniones y convencimientos

4.5 Derecho a cambiar de idea o de línea de acción

4.6 Derecho a la crítica y a protestar por un trato injusto

Sesión No. 5. Derechos Asertivos

4.7 Derecho a interrumpir para pedir una aclaración

4.8 Derecho a intentar un cambio.

4.9 Derecho a pedir ayuda o apoyo emocional

4.10 Derecho a sentir y expresar el dolor.

4.11 Derecho a ignorar los consejos de los demás

4.12 Derecho a recibir el reconocimiento formal por un trabajo bien hecho

4.13 Derecho a decir "no"

Sesión No. 6. Derechos Asertivos (tercera parte)

4.14 Derecho a estar solo aun cuando los demás deseen su compañía

4.15 Derecho a no justificarse ante los demás.

4.16 Derecho a no responsabilizarse de los problemas de los demás

4.17 Derecho a no anticiparse a las necesidades y deseos de los demás

4.18 Derecho a no estar pendiente de la buena voluntad de los demás.

4.19 Derecho a responder o a no hacerlo

Sesión No. 7. Persona asertiva

5.1 Conducta Agresiva

5.2 Conducta Pasiva

5.3 Conducta Asertiva

5.4 Perfil de la persona Asertiva

Sesión No. 8. La asertividad

Estudio de Casos

Sesión No. 9. Valoración de lo aprendido por los alumnos, a través de ejercicios

Resolución de dudas y retroalimentación

Sesión No. 10. Evaluación Final

Tabla 4 Contenido de las sesiones de la propuesta del curso-taller Fuente: Elaboración Propia

\section{Referencias}

Acosta Alberto, López Megías Jesús, Segura Ignacio, Rodríguez Amador Emiliano (s.f) Andalucía, España. universidad de granada. cuaderno de habilidades alternativas a la agresión. programa de educación para la convivencia. localización isbn 84-668-2406-2. depósito legal: gr-998/03. recuperado: 14 de septiembre

2014 http://www.juntadeandalucia.es/educacion/web portal/abaco-portlet/content/efb46430-71614811-ac21-aa182c259636

Arellano, Norka (2006). las barreras en la comunicación no verbal entre docente-alumno. revista orbis /ciencias humanas/año 2/no. 4 /2006. pág. 3.

Belmont, J.: estrategias cognoscitivas $\mathrm{y}$ aprendizaje estratégico. en acción pedagógica. vol. $2(1,2)$. 1991. p.62-63. tomado de rebollar morote alfredo (2000) tesis doctoral una variante para la estructuración del proceso de enseñanza aprendizaje de la matemática, a partir de una nueva forma de organizar el contenido, en la escuela media cubana

Bruner, J., (1972) el proceso de educación, México: ed. uteha.

Bruner, J., (1978) el proceso mental en el aprendizaje, Madrid: ed. narcea.

Bruner, J., (1984) el desarrollo de los procesos de representación, en: acción, pensamiento y lenguaje, Madrid: alianza ed.

Bruner, J., (1986) realidad mental y mundos posibles, barcelona: ed. gedisa.

Bruner, J., (1988) desarrollo cognitivo y educación, Madrid: ediciones morata.

caballo V.E. (1993) manual de evaluación y entrenamiento de las habilidades sociales., ed. siglo $\mathrm{xxi}$

Caballo Vicente E. (1983) Madrid, España. asertividad. definiciones y dimensiones. universidad autónoma de madrid. estudios de psicología no. 13, 1983. publicado en dialnet, localización: estudios de psicología, issn-02109395, issn-e 1579-3699, no. 13, 1983, págs. 52- 
Caballo, V. E. (s.f.). asertividad: definiciones y dimensiones consultado en http://dialnet.unirioja.es/servlet/articulo?codigo $=65876$. recuperado 05 septiembre 2019 .

Caballo, V. E. y Carrobles, J.A. (1988). comparación de la efectividad de diferentes programas de entrenamiento en habilidades sociales. revista española de terapia del comportamiento, 6,93-114.

Caballo, V.E. (1988). teoría, evaluación y entrenamiento de las habilidades sociales. valencia. promolibro.

Castanyer, Olga 2002, la asertividad: expresión de una sana autoestima. $17^{\circ}$ edición. Bilbao. desclée de brouwer.

Castanyer, Olga. (2008) ¿porqué no logro ser asertivo? bilbao. ed. descleé de brouwer.

Cerezal Mezquita, Julio; Fiallo Rodríguez, Jorge, 2002, ciudad de la habana, cuba. los métodos científicos en las investigaciones pedagógicas.

De Barros, Nidia Aylwin y Gissi, Bustos Jorge (s.f)., citado en centro de estudios de opinión. universidad de antioquía. Facultad de Ciencias Sociales y Humanas Op. Cit. http://aprendeenlinea.udea.edu.co/revistas/index .php/ceo/article/viewFile/1650/1302 recuperado 15 de septiembre 2014.

Delgado Arcos, Esteban; Pablos Márquez, Manuel 1; Sánchez Sánchez, domingo (2009). Andalucía, España. dino. educación preventiva sobre drogas para preadolescentes tercer ciclo de la educación primaria. núcleo temático no, 1. auto estima, habilidades sociales y toma de decisiones.

Delgado Jennifer (2013). pedir ayuda, ¿por qué nos resulta tan difícil? temática: desarrollo personal.

http://www.rinconpsicologia.com/2013/12/pedi

r-ayuda-por-que-nos-resulta-tan.html

recuperado el 22 de septiembre de 2014.

Diez Gutiérrez, enrique javier (s.f.). entrenamiento asertivo. universidad de león, españa. practicum de magisterio: especialidad educación infantil.
Garrido Pérez, Estrella de la c; Ortega Andrade, Norma Angélica; escobar torres, jorge; garcía cruz, rubén (2010) méxico. evaluacion de la asertividad en estudiantes universitarios con bajo rendimiento académico. revista científica electrónica de psicología, icsa-uaeh no. 9.

Kisnerman, Natalio. los talleres, ambientes de formación profesional. en: el taller, integración de teoría y práctica. de barros, nidia a. gissi, jorge y otros. editorial humanitas. buenos aires. 1977.

León, A., Rodríguez, C., Ferre, f. y Ceballos, G. (2009). asertividad y autoestima en estudiantes de primer semestre de la facultad de ciencias de la salud de una universidad pública de la ciudad de santa marta (colombia). revista psicología desde el caribe, 24, 91 - 105.

Marcuello García, Ángel Antonio (s.f.) ferrol la coruña, España. la asertividad, técnicas para decir "no" al consumo de drogas. http://www.psicologia-

online.com/autoayuda/asertividad/decir_no_a_1 as_drogas.shtml recuperado 22 de septiembre de 2014.

Martínez de Velazco, a., Nosnik, a. (2004) comunicación organizacional práctica, manual gerencial, México: trillas.

Martínez, M. (1999). el enfoque sociocultural en el estudio del desarrollo y la educación. revista electrónica de investigación educativa, 1 (1). consultado el día 16 de septiembre de 2014 en: http://redie.uabc.mx/vol1no1/contenidomtzrod.html

Mirabent Perozo, gloria. revista pedagógica cubana. año ii abril - junio no. 6. la habana, 1990 http://aprendeenlinea.udea.edu.co/revistas/index .php/ceo/article/viewfile/1650/1302

Palacio, J. y Martínez, y. (2007). relación del rendimiento académico con la salud mental en jóvenes universitarios. revista psicogente, 10, $113-128$.

Palacio, J., Martínez, y., Ochoa, N. y Tirado, E. (2006). relación del rendimiento académico con las aptitudes mentales, salud mental, autoestima y relaciones de amistad en jóvenes universitarios de atlántico y bolívar. revista psicogente, 9,1131. 
Real Academia Española. diccionario de la lengua española (drae) es la obra de referencia de la academia. la edición actual -la 22. ${ }^{\text {, }}$, publicada en 2001. http://lema.rae.es/drae/?val=curso recinto de quirama; mimeo.

Rebollar Morote Alfredo (2000) tesis doctoral una variante para la estructuración del proceso de enseñanza aprendizaje de la matemática, a partir de una nueva forma de organizar el contenido, en la escuela media cubana

Reyes, Gómez Melba. (s.f) citado en centro de estudios de opinión. universidad de antioquía. facultad de ciencias sociales y humanas. el taller en trabajo social. en: kisnerman, n. op. cit. $\mathrm{http}: / /$ aprendeenlinea.udea.edu.co/revistas/index .php/ceo/article/viewfile/1650/1302 recuperado 15 septiembre 2014.

Reyes, Y.N. (2003). relación entre el rendimiento académico, la ansiedad ante los exámenes, los rasgos de personalidad, el autoconcepto y la asertividad en estudiantes de primer año de psicología de unms. tesis de pregrado en psicología de unms. facultad de psicología, lima-perú.

Roca, Elia; (s.f.) programa de autoestima, asertividad e inteligencia emocional, http://www.cop.es/colegiados/cv00520/hs_1\%c $2 \%$ aa\%20sesion.pdf recuperado 14 septiembre 2014.

Rodríguez Palmero, Ma. Luz; (2008) Barcelona. la teoría del aprendizaje significativo en la perspectiva de la psicología cognitiva. editorial octaedro. edición electrónica.

Secretaría de Educación Pública (2013) México. plan sectorial de educación 2013-2018. primera edición.

Trejo Rayón, Fernando (2006), comunicación asertiva. cap. 7. conductas pasiva, agresiva y asertiva. publicado el 24 de febrero de 2006. recuperado 14 de septiembre de 2014. http://www.mailxmail.com/curso-

comunicacion-asertiva/conductas-pasivaagresiva-asertiva
Universia. México. (2011) sitio de noticias. educación superior.

http://noticias.universia.net.mx/vidauniversitaria/noticia/2011/06/02/832742/preocu pa-falta-valores-jovenes-institucioneseducacion-superior.html recuperado 02 oct 2014.

Vigotsky (1999). citado por Martínez (1999) en el enfoque sociocultural en el estudio del desarrollo y la educación. revista electrónica de investigación educativa, 1 (1). consultado el día 16 de septiembre de 2014 en: http://redie.uabc.mx/vol1no1/contenidomtzrod.html

Wertsch, (1985; 1989; 1991); Karmiloff-Smith, (1979) citado por Martínez (1999) en el enfoque sociocultural en el estudio del desarrollo y la educación. revista electrónica de investigación educativa, 1 (1). recuperado el 16 de septiembre de 2014

http://redie.uabc.mx/vol1no1/contenidomtzrod.html

Zilberstein Toruncha, José, Silvestre Oramas, Margarita, 2008. antología curso de tutores de tesis. universidad tangamanga. 